\title{
Trajectory optimization for the Horyu-VI international lunar mission
}

\author{
Federico De $\operatorname{Grossi}^{1}(\bowtie)$, Paolo Marzioli ${ }^{1}$, Mengu Cho ${ }^{2}$, Fabio Santoni $^{1}$, and Christian Circi ${ }^{1}$
}

1. Sapienza University of Rome, 00138 Rome, Italy

2. Kyushu Institute of Technology, Kitakyushu 804-8550, Japan

\section{ABSTRACT}

The Horyu-VI nano-satellite is an international lunar mission with the purpose of studying the lunar horizon glow (LHG) — a still unclear phenomenon caused by electrostatically charged lunar dust particles. This study analyzes the mission trajectory with the hypothesis that it is launched as a secondary payload of the NASA ARTEMIS-II mission. In particular, the effect of the solar gravity gradient is studied; in fact, depending on the starting relative position of the Moon, the Earth, and the Sun, the solar gradient acts differently on the trajectory - changing it significantly. Therefore, the transfer and lunar capture problem is solved in several cases with the initial Sun-Earth-Moon angle as the key parameter. Furthermore, the inclination with respect to the Moon at capture is constrained to be equatorial. Finally, the problem of stabilization and circularization of the lunar orbit is addressed in a specific case, providing an estimate of the total propellant cost to reach the final orbit around the Moon.
\end{abstract}

\author{
KEYWORDS \\ trajectory optimization \\ Artemis mission \\ lunar mission \\ lunar horizon glow (LHG) \\ weak stability boundary
}

\section{Research Article}

Received: 30 April 2021

Accepted: 2 July 2021

(C) The Author(s) 2021

\section{Introduction}

The Horyu-VI mission is a nano-satellite lunar mission developed by the Kyushu Institute of Technology, in collaboration with the Nanyang Technological University of Singapore, Sapienza University of Rome, and the California Polytechnic State University [1]. The mission was proposed as a secondary payload of the ARTEMIS-II NASA mission.

The purpose of Horyu-VI is to study the lunar horizon glow (LHG) phenomenon. It appears as a slim brightness above the lunar surface, and was observed by the astronauts of the Apollo missions and pictures of it were taken by Surveyor 1, 5, 6, and 7. It is believed that the LHG is caused by lunar dust particles scattering light at sunrise and sunset; and since the brightness was stronger than the level that could be produced by micrometeorite ejecta, a mobilized dust population by electrostatic forces was predicted to be responsible for the forward scattering of sunlight [1-12].

To date, there has been no dedicated mission to observe the lunar horizon glow, especially near and be- low a 1-km altitude. A small satellite platform can be used to continuously monitor the forward-scattering of sunlight above the lunar terminator region to detect the column density of the lunar dust cloud. The most recent attempt to detect the lunar horizon glow came from the lunar orbiter laser altimeter and the laser ranging telescope onboard the Lunar Reconnaissance Orbiter [2]. These measurements aimed to test the hypothesis of a major meteor stream producing sufficient ejecta for the LHG. The outcome is most likely that the LHG is a rare occurrence at altitudes below $20 \mathrm{~km}$ during meteor streams. These measurements could not put any constraint for the LHG near a 1-km altitude and below - where most of the exploration activities are present. The study of the LHG could reveal critical information about the dust particles near the surface and at low altitudes - information which could be important for future human and robotic missions since the regolith dust is a significant risk factor in the lunar environment [13-18]. A low lunar orbit is the most promising candidate for studying the LHG, from where 


\section{Nomenclature}

\begin{tabular}{|c|c|}
\hline$a$ & semi-major axis $(\mathrm{km})$ \\
\hline$e$ & eccentricity \\
\hline$i$ & inclination $\left({ }^{\circ}\right)$ \\
\hline$\Omega$ & $\operatorname{RAAN}\left({ }^{\circ}\right)$ \\
\hline$\omega$ & argument of pericenter $\left(^{\circ}\right)$ \\
\hline$\theta$ & true anomaly $\left({ }^{\circ}\right)$ \\
\hline$r$ & spacecraft position vector $(\mathrm{km})$ \\
\hline $\boldsymbol{v}$ & spacecraft velocity vector $(\mathrm{km} / \mathrm{s})$ \\
\hline $\boldsymbol{r}_{\mathrm{m}}$ & position vector from the Moon to the spacecraft $(\mathrm{km})$ \\
\hline $\boldsymbol{R}_{\mathrm{m}}$ & Moon position vector $(\mathrm{km})$ \\
\hline$r_{\mathrm{s}}$ & position vector from the Sun to the spacecraft $(\mathrm{km})$ \\
\hline $\boldsymbol{R}_{\mathrm{S}}$ & Sun position vector $(\mathrm{km})$ \\
\hline $\boldsymbol{F}$ & thrust of the spacecraft $(\mathrm{N})$ \\
\hline$m$ & mass of the spacecraft $(\mathrm{kg})$ \\
\hline$I_{\mathrm{sp}}$ & specific impulse $(\mathrm{s})$ \\
\hline$\mu_{\mathrm{E}}$ & Earth gravitational constant $\left(\mathrm{km}^{3} / \mathrm{s}^{2}\right)$ \\
\hline$\mu_{\mathrm{M}}$ & Moon gravitational constant $\left(\mathrm{km}^{3} / \mathrm{s}^{2}\right)$ \\
\hline$\mu_{\mathrm{S}}$ & Sun gravitational constant $\left(\mathrm{km}^{3} / \mathrm{s}^{2}\right)$ \\
\hline$\theta_{\mathrm{sm} 0}$ & Sun-Earth-Moon angle at the initial time $\left(^{\circ}\right)$ \\
\hline$J$ & cost function \\
\hline$t_{\mathrm{f}}$ & time of flight (day) \\
\hline$m_{\mathrm{p}}$ & mass of propellant $(\mathrm{kg})$ \\
\hline$\alpha, \beta$ & angles of the thrust-variables of optimization $\left({ }^{\circ}\right)$ \\
\hline$N_{\text {arc }}$ & number of arcs of a trajectory \\
\hline$h_{\mathrm{p}}$ & lunar pericenter height $(\mathrm{km})$ \\
\hline$h_{\text {flyby }}$ & height of lunar flyby (km) \\
\hline $\boldsymbol{a}_{\mathrm{SRP}}$ & solar radiation pressure acceleration vector $\left(\mathrm{m} / \mathrm{s}^{2}\right)$ \\
\hline$A$ & spacecraft area $\left(\mathrm{m}^{2}\right)$ \\
\hline$P_{\mathrm{E}}$ & solar radiation pressure at $1 \mathrm{AU}\left(\mathrm{N} / \mathrm{m}^{2}\right)$ \\
\hline
\end{tabular}

it is also possible, in principle, to extend the scientific objectives by releasing femto-satellites - such as smart dust from the satellite [19].

The present work focuses on the trajectory analysis of the Horyu mission: the transfer trajectory, in particular the effect that the solar gravity gradient has on it, and the stabilization of the orbit around the Moon.

The spacecraft is considered equipped with low-thrust Hall effect thrusters, whose acceleration is smaller than the Sun gravity gradient for most of the time during the transfer trajectory to the Moon; therefore, the effect of the Sun is of great importance and, according to the initial position of the Earth and the Moon with respect to the Sun, the trajectory changes significantly. The trajectory is computed in several cases, covering all the variation range of the Sun-Earth-Moon angle, which is the main parameter that changes the effect of the solar gradient on the trajectory.

The paper is composed of the following sections:

Section 2 presents the spacecraft architecture and main design features. In Section 3, the strategy employed for the design and optimization of the trajectory is described.
In Section 4, the obtained results are presented and analyzed, and a constraint on the final inclination at the arrival at the Moon is considered, as well as its influence on the trajectory. Section 5 presents further analysis on the sensitivity of the trajectory to small changes in the initial position of the Moon, affecting the flyby at the beginning of the trajectory, as well as an estimate of the effect of the solar radiation pressure.

In Section 6, the problem of stabilization and circularization of the orbit is considered for one of the obtained trajectories. The conclusions are finally presented in Section 7.

\section{Horyu-VI spacecraft architecture and design features}

The Horyu-VI nano-satellite is a $12 \mathrm{U}$ CubeSat (approximately $200 \mathrm{~mm} \times 200 \mathrm{~mm} \times 300 \mathrm{~mm}$ ) conceived for LHG observations in a lunar trajectory [1]. The spacecraft architecture is illustrated in Fig. 1. The mission was conceived for NASA's ARTEMIS-II call for secondary payload proposals issued in 2019. The CubeSat payload 


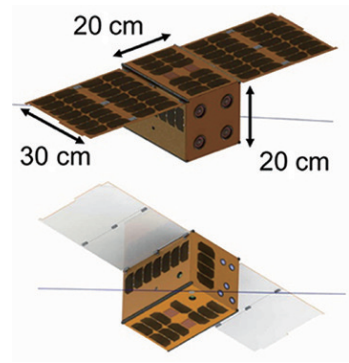

Fig. 1 CAD image of Horyu-VI.

is composed of imaging sensors at different wavelengths (monochrome, RGB, near-infrared (NIR) and ultraviolet (UV) to detect and investigate LHG in lunar orbit. The spacecraft navigation will be made possible by groundbased range and range rate determination. The on-board transponder uses a chip-sized atomic clock (CSAC) for time reference. The cis-lunar space characterization of the behavior of such a time-keeping device, whose operations were already in-orbit demonstrated through the SPATIUM-I mission in low Earth orbit (LEO), can be considered a secondary objective for spacecraft missions. The spacecraft propulsion system relies on four xenon gas Hall effect micro-thrusters capable of providing a thrust of $150 \mu \mathrm{N}$ each. The thrusters are located on the same side of the spacecraft, providing a total thrust of $600 \mu \mathrm{N}$. The required electrical power for the maneuvers, approximately $60 \mathrm{~W}$ for the entire system, will be generated by the spacecraft deployable solar panels and stored by the on-board batteries. The total amount of stored gas propellant is approximately $3 \mathrm{~kg}$; and the dedicated CubeSat volume to the propulsion and propellant storage subsystems was approximately 8 units. The spacecraft propulsion system does not involve any thrust vectoring systems, so the thrust vector direction is controlled by the attitude determination and control subsystem. This will rely on reaction wheels for fine attitude control and on cold-gas thrusters, using xenon and sharing the propellant tanks with the propulsion system for de-saturation. The main attitude determination sensors are optical star trackers, and inertial measurement units. The entire system will assure a 0.1 degree pointing accuracy.

\section{Trajectory design}

As stated above, the mission begins as a secondary payload of the SLS rocket in an Artemis mission. The SLS upper stage can release the secondary payloads on three bus stops along its disposal trajectory at altitudes of
$36,500,70,000$, and 93,500 km [20]. In this mission analysis, it is assumed that the spacecraft is released at an altitude of $70,000 \mathrm{~km}$ on the upper stage disposal trajectory. If no thrust is applied, the spacecraft encounters the Moon on a flyby - which increases its energy; therefore, without control, the spacecraft will abandon the Earth-Moon system.

The initial conditions for our simulation are in reference to December 15, 2017-14:56:42.2 TDB date; and the trajectory is propagated considering the Earth-MoonSun restricted four-body problem dynamics. The initial orbital elements, referred to as the Earth, are given in Eq. (1); and the equations of motion of the spacecraft in a Cartesian Earth-centered inertial reference frame are given in Eq. (2).

$$
\begin{aligned}
& \left\{\begin{array}{l}
a=206076.92 \mathrm{~km}, e=0.9667, i=28.61^{\circ} \\
\Omega=65.96^{\circ}, \omega=47.92^{\circ}, \theta_{0}=148.41^{\circ}
\end{array}\right. \\
& \left\{\begin{aligned}
\dot{\boldsymbol{r}}= & \boldsymbol{v} \\
\dot{\boldsymbol{v}}= & \boldsymbol{g}_{\mathrm{E}}+\boldsymbol{g}_{\mathrm{pM}}+\boldsymbol{g}_{\mathrm{pS}}+\boldsymbol{a}_{\mathrm{c}}=-\frac{\mu_{\mathrm{E}}}{r^{3}} \boldsymbol{r} \\
& -\mu_{\mathrm{M}}\left(\frac{\boldsymbol{r}_{\mathrm{m}}}{r_{\mathrm{m}}^{3}}+\frac{\boldsymbol{R}_{\mathrm{m}}}{R_{\mathrm{m}}^{3}}\right)-\mu_{\mathrm{S}}\left(\frac{\boldsymbol{r}_{\mathrm{s}}}{r_{\mathrm{s}}^{3}}+\frac{\boldsymbol{R}_{\mathrm{s}}}{R_{\mathrm{s}}^{3}}\right)+\frac{\boldsymbol{F}}{m}
\end{aligned}\right.
\end{aligned}
$$

where $\boldsymbol{r}, \boldsymbol{v}$ are the position and velocity vectors respectively, $\boldsymbol{g}_{\mathrm{E}}$ is the gravitational acceleration of the Earth, $\boldsymbol{g}_{\mathrm{pM}}$ is the gravity effect from the Moon, $\boldsymbol{g}_{\mathrm{pS}}$ is the gravity effect from the Sun; $\boldsymbol{a}_{\mathrm{c}}$ is the thrust acceleration, $\mu_{\mathrm{E}}, \mu_{\mathrm{M}}$, and $\mu_{\mathrm{S}}$ are the Earth, the Moon, and the Sun gravitational parameters, respectively; $\boldsymbol{r}_{\mathrm{m}}$ is the vector from the Moon to the spacecraft; $\boldsymbol{R}_{\mathrm{m}}$ is the vector from the Earth to the Moon; $\boldsymbol{r}_{\mathrm{s}}$ is the vector from the Sun to the spacecraft; $\boldsymbol{R}_{\mathrm{s}}$ is the vector from the Earth to the Sun; $\boldsymbol{F}$ is the thrust vector; and finally, $m$ is the mass.

The spacecraft is supposed to have an initial mass $m_{0}=20 \mathrm{~kg}$, a total thrust of $F=600 \mu \mathrm{N}$, and a specific impulse of $I_{\mathrm{sp}}=1000 \mathrm{~s}$.

The final orbit around the Moon is a circular, equatorial low lunar orbit with a height of $100 \mathrm{~km}$, and there are no further constraints on the right ascension or the argument of the pericenter. For the purpose of this study, the orbit to be achieved can be prograde or retrograde, with a margin of $30^{\circ}$ from the conditions of the equatorial orbit. In the following section, only the transfer trajectory is addressed, with the objective of obtaining an orbit with eccentricity less than one, and pericenter height equal to $200 \mathrm{~km}$. Initially, the inclination of the arrival orbit 
around the Moon was left as free, and then the difference was shown when it was constrained instead.

Different launching dates for the mission imply different Sun-Moon relative initial positions; therefore, the solar gradient affects the trajectory in different wayschanging the cost of the trajectory in terms of time and propellant needed. To analyze these effects, starting from the configuration of the date to which the initial conditions are referred to, the position of the Sun is moved by intervals of $10^{\circ}$ of the true anomaly along its apparent orbit around the Earth, until a complete turn is completed. This generates new configurations of the Sun-Earth-Moon system, which can be described by the Sun-Earth-Moon angle at the initial time $\theta_{\mathrm{sm} 0}$; and the trajectory is therefore computed in all 36 configurations of $\theta_{\mathrm{sm} 0}$.

$$
\theta_{\mathrm{sm} 0}= \pm \arccos \left(\widehat{\boldsymbol{R}}_{\mathrm{m} 0} \cdot \widehat{\boldsymbol{R}}_{\mathrm{s} 0}\right)
$$

The Sun-Earth-Moon angle is computed as in Eq. (3), and the plus sign is taken for the anti-clockwise rotation of $\widehat{\boldsymbol{R}}_{\mathrm{s} 0}$ from $\widehat{\boldsymbol{R}}_{\mathrm{m} 0}$, and the minus otherwise. Figure 2 shows the angle, showing the orbital planes of the Moon and Sun in the Earth-centered frame.

The problem is approached as an optimization problem. The trajectory is divided in $N$ arcs of duration $\Delta t_{i}$, during which the thrust direction is fixed in the inertial frame and defined by two angles $\alpha_{i}$ and $\beta_{i}$, and the number of optimization variables is therefore $3 N$.

$$
\begin{aligned}
\boldsymbol{F}_{i} & =F_{\text {nom }}\left(\cos \alpha_{i} \cos \beta_{i} \quad \sin \alpha_{i} \cos \beta_{i} \sin \beta_{i}\right)^{\mathrm{T}} \\
\boldsymbol{u}_{\mathrm{opt}} & =\left(\Delta t_{1} \Delta t_{2} \cdots \Delta t_{N}, \alpha_{1} \beta_{1} \alpha_{2} \beta_{2} \cdots \alpha_{N} \beta_{N}\right)^{\mathrm{T}} \\
J & =t_{\mathrm{f}}+C
\end{aligned}
$$

Equation (4) is the thrust vector in the inertial geocentric frame during the $i$ th arc; Eq. (5) shows the vector of optimization variables; Eq. (6) is the cost function, where $t_{\mathrm{f}}=\sum_{i=1}^{N} \Delta t_{i}$ is the duration of the trajectory, and $C$ is the constraint on the final state as penalty functions. No coasting arcs are considered; therefore, if the $t_{\mathrm{f}}$ is minimum, the propellant mass is also minimum.

The design method of the trajectory is as follows: a first starting guess is obtained by manually choosing the variables, and then an optimization is run with $C$ in the cost function in the form of Eq. (7) - where $r_{j}$ and $R_{\mathrm{m}_{j}}$ are the components of the position vector of the spacecraft and of the Moon in the geocentric frame respectively. $v_{j}$ and $V_{\mathrm{m}_{j}}$ are the components of the velocity vector of the spacecraft and of the Moon in the geocentric frame respectively, as in common rendezvous trajectory approaches [21]. The first optimization aims to find a trajectory in which the spacecraft encounters the Moon. However, it is not sufficient to obtain the orbit with the required pericenter height as it often yields final conditions with non-optimal semi-axis and eccentricity - which can be improved. Therefore, another optimization is run with $C$ as shown in Eq. (8), where $h_{\mathrm{p}}$ is the pericenter height over the Moon, $h_{\mathrm{t}}=200 \mathrm{~km}$, and $e_{\mathrm{m}}$ and $a_{\mathrm{m}}$ are the final eccentricity and semi-axis with respect to the Moon respectively. The optimization is repeated, progressively reducing $a_{\mathrm{t}}$ and $e_{\mathrm{t}}$, the target semi-axis and eccentricity around the Moon respectively, until $a_{\mathrm{m}}$ and $e_{\mathrm{m}}$ no longer decrease, with $e_{\mathrm{m}}<1$ and $h_{\mathrm{p}}=h_{\mathrm{t}}$.

$$
C=c_{R_{\mathrm{m}}} \sum_{j=1}^{3}\left|r_{j}-R_{\mathrm{m}_{j}}\right|+c_{V_{\mathrm{m}}} \sum_{j=1}^{3}\left|v_{j}-V_{\mathrm{m}_{j}}\right|
$$

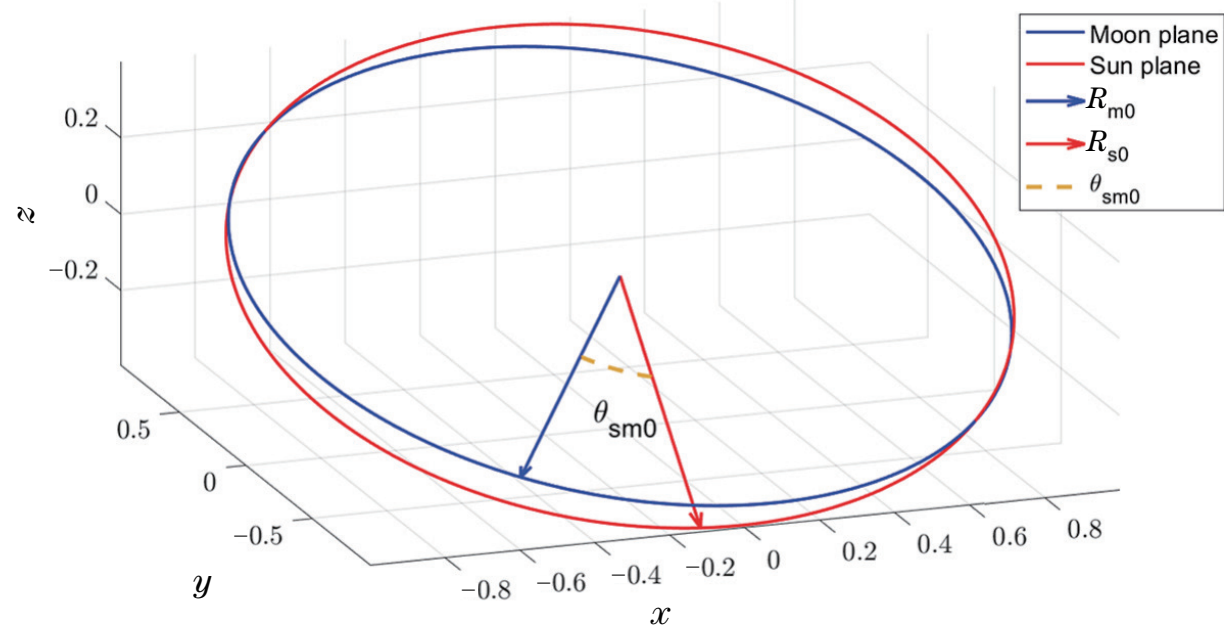

Fig. 2 Visualization of the Sun-Earth-Moon angle $\theta_{\operatorname{sm} 0}$. 


$$
C=c_{h_{\mathrm{p}}}\left|h_{\mathrm{p}}-h_{\mathrm{t}}\right|+c_{e}\left|e_{\mathrm{m}}-e_{\mathrm{t}}\right|+c_{a}\left|a_{\mathrm{m}}-a_{\mathrm{t}}\right|
$$

The employed optimizers are the interior-point algorithm of the MATLAB function fmincon; the constraints considered are only the boundaries: $\Delta t_{i, \min }=$ $0, \Delta t_{i, \max }=400$ days, $\alpha_{i, \min }=-180^{\circ}, \alpha_{i, \max }=180^{\circ}$, $\beta_{i, \min }=-90^{\circ}, \beta_{i, \max }=90^{\circ}$; the remaining options were set as default, except for the maximum function evaluations, which were set at 4000 . The Nelder-Mead algorithm of the function fminsearch was used as well, with default options except the maximum number of function evaluations, which were set at 3000 . The MATLAB version used was R2019a.

\section{Discussion on the results}

The exploitation of the solar gradient to obtain low-cost Earth-Moon transfers is employed in the weak stability boundary (WSB) or Belbruno lunar trajectories. In these trajectories, the gravity gradients of the Sun and the Earth are used to modify the trajectory, raising the perigee in the intermediate phase and decelerating in the final phase to obtain a ballistic capture around the Moon [22-24]. WSB trajectories were used in the EarthMoon transfers by the JAXA Hiten mission (1990) for the first time, as suggested by Edward Belbruno and later by the NASA GRAIL mission (2011). A similar strategy can be exploited in our case where we are trying to obtain the best effect from the Sun and the Earth to achieve a capture similar to the one typical of WSB. In contrast to the classical ballistic WSB trajectory in our problem, there is also a flyby, and low-thrust propulsion is used.

After the lunar flyby, the spacecraft needs to be decelerated-but the thrust alone is not sufficient to do this, especially if the solar gradient accelerates instead. The analysis showed that the most efficient trajectories have an initial phase before the apogee in which the solar gradient decelerates the spacecraft, and a phase after the apogee where it accelerates, easing the increase of the perigee. This class of trajectories are the best in terms of time of flight and propellant among the ones found, and appear to be possible for values approximately between $\theta_{\mathrm{sm} 0} \in\left[-30^{\circ}, 0^{\circ}\right]$, and between $\theta_{\mathrm{sm} 0} \in\left[160^{\circ}, 180^{\circ}\right]$-with a duration between 81 and 141 days. Figure 3 shows one such trajectory in the geocentric reference frame, the thrust (blue arrows) and the gradient (red arrows) are shown; the red dashed line represents the scaled path of the Sun during the trajectory. The trajectory showed is the best among the ones found; it has a $t_{\mathrm{f}}=81.5$ days, a mass of propellant required of $0.43 \mathrm{~kg}$, and it achieves a lunar orbit with an eccentricity of 0.93 , and the value of

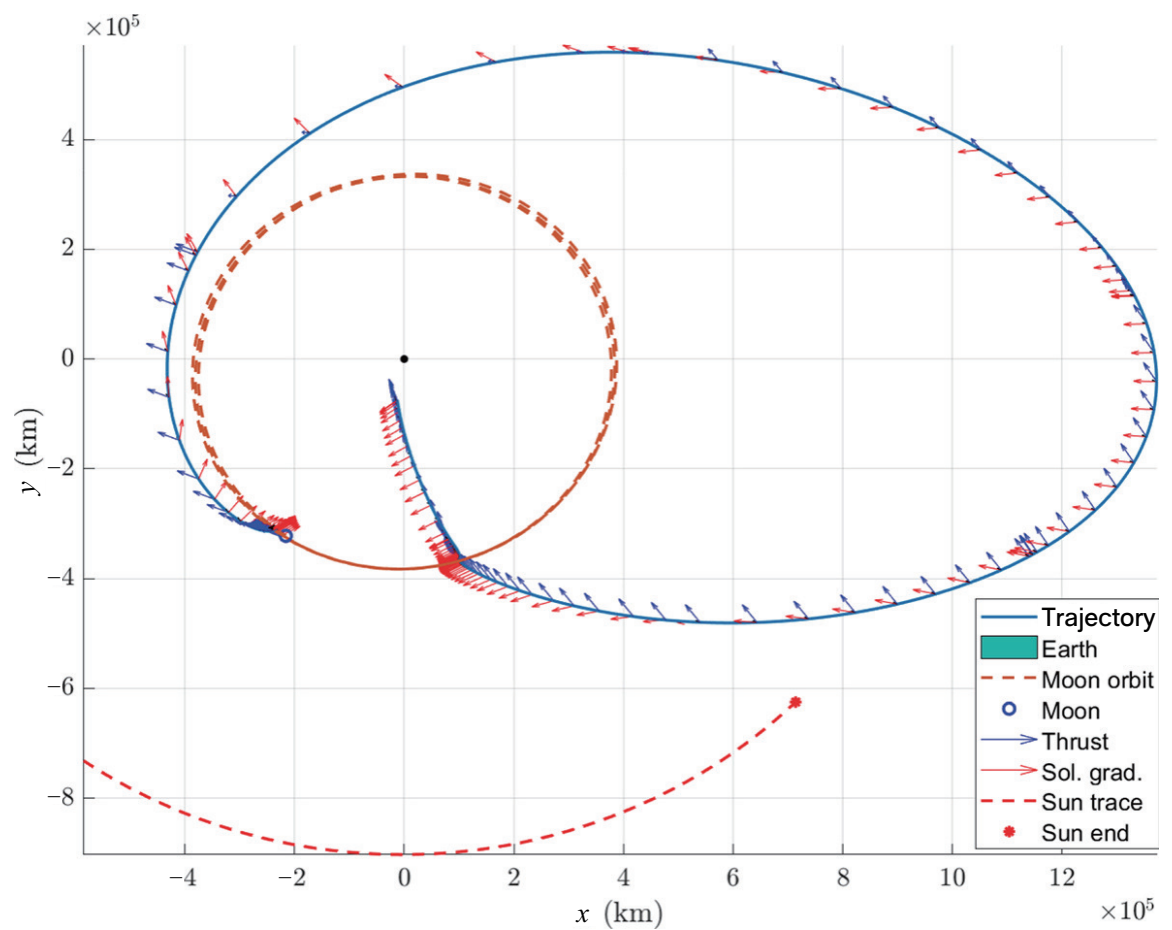

Fig. 3 WSB-like trajectory in the Earth-centred reference frame, $\theta_{\mathrm{sm} 0}=-5.78^{\circ}$.

(10) TNIIVRSITY PRESA 
the Sun-Earth-Moon angle is equal to $\theta_{\mathrm{sm0}}=-5.78^{\circ}$.

Figure 4 shows the same trajectory in the Sun-Earth co-rotating frame in the left plot. In this reference frame, the direction of the Sun is constant; thus, the gradient acceleration field does not change - it is represented by the red arrows in the plot. The right plot shows the arrival at the Moon. It can be seen that the Earth's gravity gradient decelerates the spacecraft in this final phase, helping during the capture. The expressions of the gradient accelerations of the Sun and Earth plotted in Figs. 3 and 4 are given in Eq. (9):

$$
\boldsymbol{g}_{\mathrm{S}}=-\mu_{\mathrm{S}}\left(\frac{\boldsymbol{r}_{\mathrm{s}}}{r_{\mathrm{s}}^{3}}+\frac{\boldsymbol{R}_{\mathrm{s}}}{R_{\mathrm{s}}^{3}}\right), \boldsymbol{g}_{\mathrm{E}}=-\mu_{\mathrm{E}}\left(\frac{\boldsymbol{r}_{\mathrm{e}}}{r_{\mathrm{e}}^{3}}+\frac{\boldsymbol{R}_{\mathrm{e}}}{R_{\mathrm{e}}^{3}}\right)
$$

As before, $\boldsymbol{r}_{\mathrm{s}}$ and $\boldsymbol{r}_{\mathrm{e}}$ are the vectors from the Sun to the spacecraft and from the Earth to the spacecraft, respectively; and $\boldsymbol{R}_{\mathrm{S}}, \boldsymbol{R}_{\mathrm{e}}$ are the vectors from the Earth to the Sun and from the Moon to the Earth, respectively. In Fig. 3, the gradient is given by the expression on the left in Eq. (9) in the Earth-centered frame, where $\boldsymbol{R}_{\mathrm{S}}$ varies according to the motion of the Sun with respect to the Earth. In Fig. 4 on the left, $\boldsymbol{g}_{\mathrm{S}}$ is computed in a reference frame with the $x$-axis parallel to the EarthSun direction; therefore, $\boldsymbol{R}_{\mathrm{S}}$ is constant in this frame. In Fig. 4 on the left, the Earth gradient is given by the expression on the right in Eq. (9) in a Moon-centered non-rotating frame, where $\boldsymbol{R}_{\mathrm{e}}$ rotates according to the motion of the Earth with respect to the Moon.

Figure 5 shows a comparison plot of the norm of the thrust acceleration and the norm of the Sun gradient acceleration during the trajectory of Figs. 3 and 4 . It can be seen that the solar gradient is a dominating acceleration in the central part of the trajectory, which is the longest part of it.

For values of $\theta_{\mathrm{sm} 0}$ farther from the most favorable ones, the trajectories have a farther apogee because the Sun gradient decelerates less after the flyby; consequently, the trajectory requires more time and more thrust effort to avoid escaping the Earth at the apogee. Trajectories like Fig. 6 can be found, presenting an unstable behavior at the apogee, and the angles of these solutions are the limit values for which WSB-like trajectories are found, after which the resemblance with WSB trajectories disappears.

In the cases where the Sun gradient accelerates the spacecraft after the flyby, the thrust is not sufficient to avoid the geocentric energy becoming greater than zero; in these cases, the duration of the trajectory increases significantly, and the thrust can be used to allow the spacecraft to re-encounter the Earth in a favorable condition for the Moon rendezvous. An example is shown in Fig. 7, where the entire trajectory is shown in the left plot, and it is zoomed on the Earth in the right plot to better show the departure and arrival.
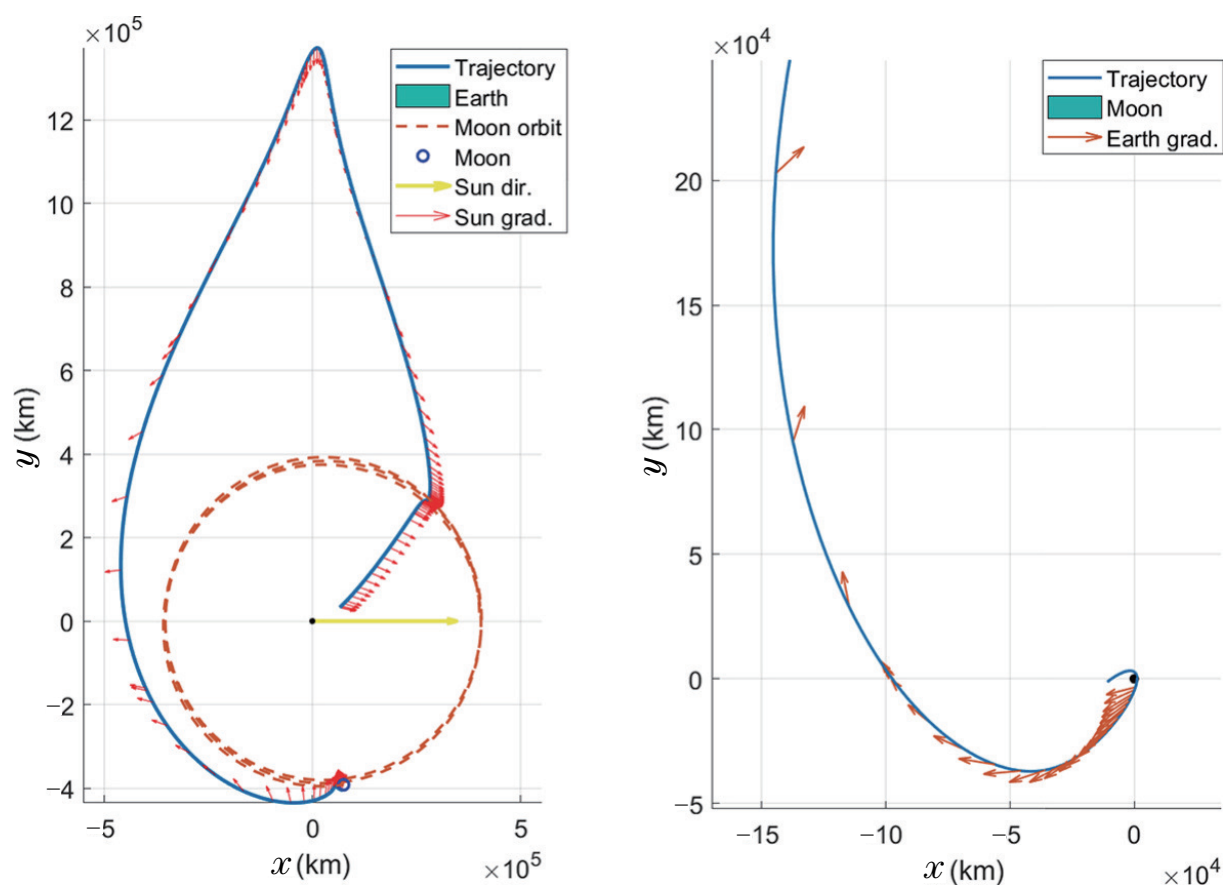

Fig. 4 WSB-like trajectory in the Sun-Earth rotating frame and at the arrival at the Moon. 


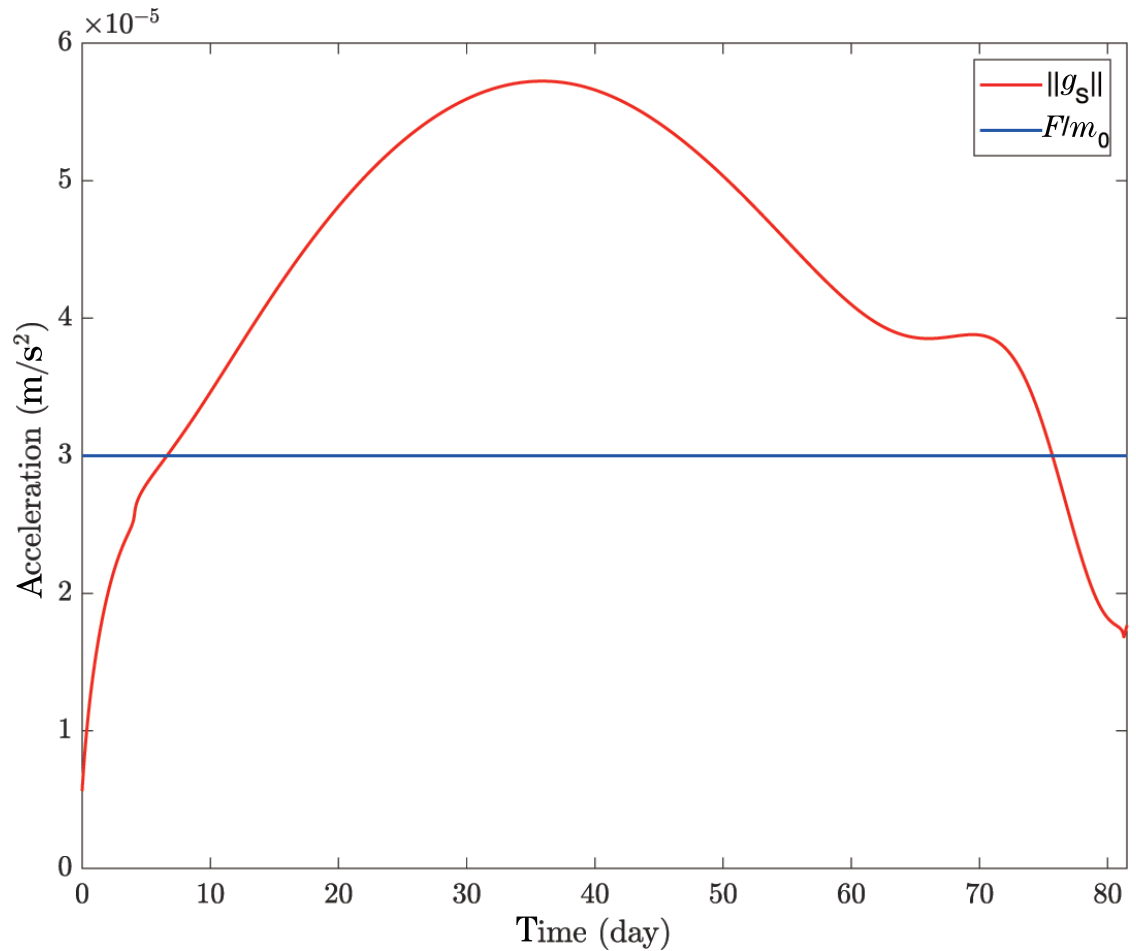

Fig. 5 Norm of the solar gradient acceleration and norm of the thrust acceleration during the trajectory of Fig. 3.

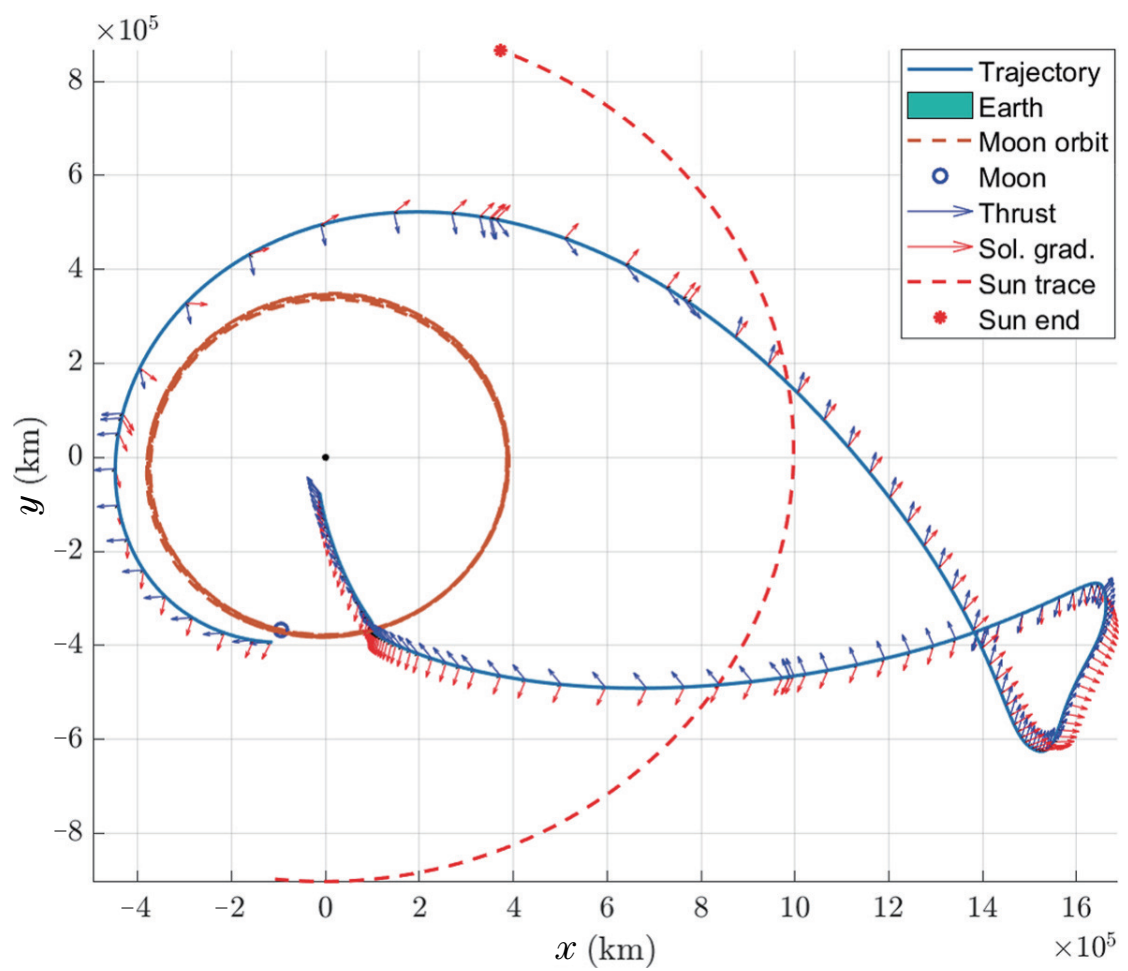

Fig. 6 Trajectory with $\theta_{\mathrm{sm} 0}=27.5^{\circ}, t_{\mathrm{f}}=165.9$ days. Earth-centered inertial reference frame.

The values of the optimization variables for the three trajectories presented above are listed in Tables 1, 2, and 3 .

Overall, for all the values of $\theta_{\mathrm{sm} 0}$ considered, it was possible to find a trajectory that achieved a close orbit around the Moon; the fastest trajectory is the one in Fig. 1 for $\theta_{\mathrm{sm} 0}=-5.78^{\circ}$ with $t_{\mathrm{f}}=81.5$ days and $m_{\mathrm{p}}=$ $0.43 \mathrm{~kg}$. The longest trajectory has $t_{\mathrm{f}}=1038.8$ days, and 

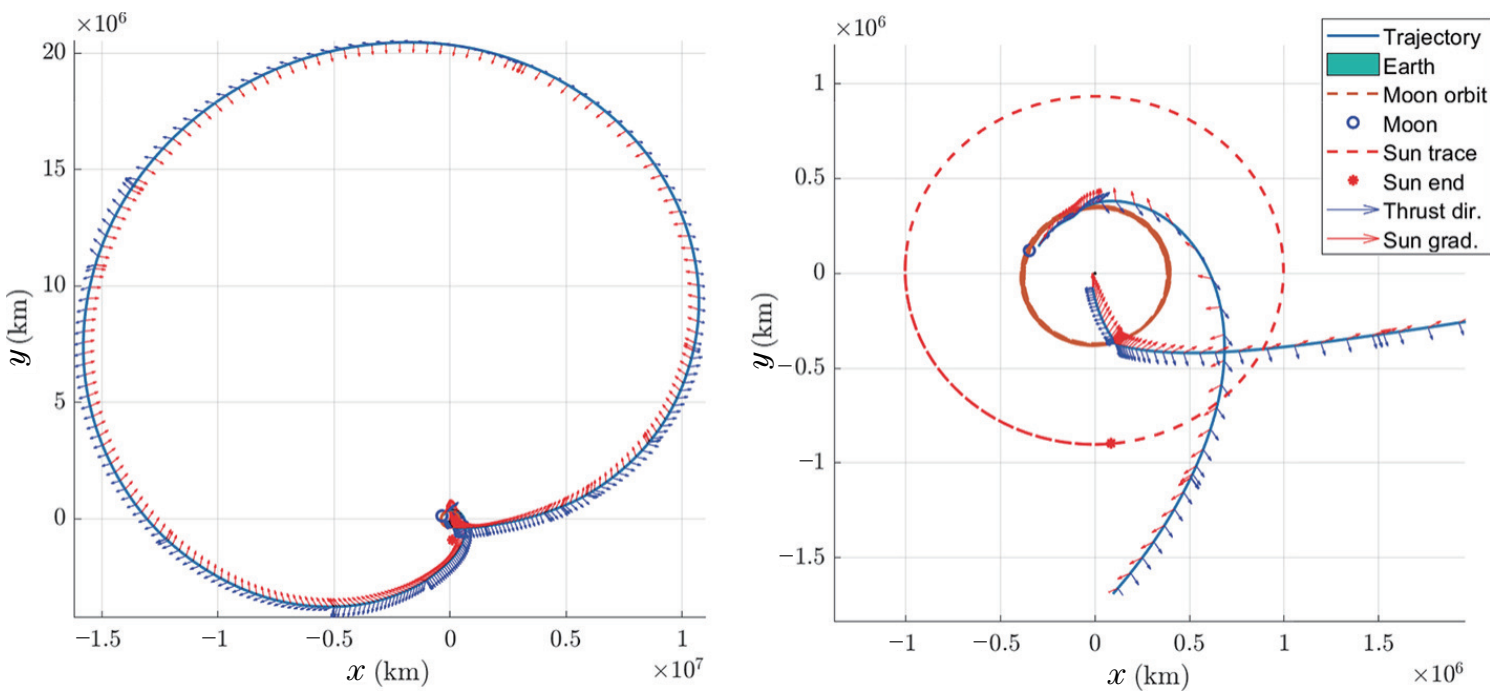

Fig. 7 Trajectory with $\theta_{\mathrm{sm} 0}=-63^{\circ}, t_{\mathrm{f}}=468.1$ days. Earth-centered inertial reference frame.

Table 1 Design variable values for the trajectory with $\theta_{\mathrm{sm} 0}=-5.78^{\circ}$

\begin{tabular}{cccc}
\hline Number of arcs & $\Delta t($ day $)$ & $\alpha(\operatorname{deg})$ & $\beta(\operatorname{deg})$ \\
\hline 1 & 2.849 & 112.106 & 11.059 \\
2 & 18.899 & 129.573 & 0.563 \\
3 & 19.989 & 124.637 & 0.009 \\
4 & 22.069 & 131.268 & 48.833 \\
5 & 10.607 & 172.478 & 69.616 \\
6 & 7.126 & 159.338 & 0.099 \\
\hline
\end{tabular}

Table 2 Design variable values for the trajectory with $\theta_{\mathrm{sm} 0}=27.5^{\circ}$

\begin{tabular}{crrr}
\hline Number of arcs & $\Delta t($ day $)$ & \multicolumn{1}{c}{$\alpha(\mathrm{deg})$} & $\beta(\mathrm{deg})$ \\
\hline 1 & 16.886 & 129.248 & 21.155 \\
2 & 11.009 & 113.292 & 27.056 \\
3 & 21.104 & -104.280 & 18.406 \\
4 & 42.540 & 51.244 & 48.687 \\
5 & 49.601 & 73.611 & 0.435 \\
6 & 6.459 & -53.544 & 7.504 \\
7 & 10.650 & -77.522 & -4.959 \\
8 & 7.699 & -176.076 & 5.307 \\
\hline
\end{tabular}

$m_{\mathrm{p}}=5.47 \mathrm{~kg}$, and it is found for $\theta_{\mathrm{sm} 0}=67.21^{\circ}$. The trajectories have a number of $\operatorname{arcs} N$ between 6 and 25 . The best trajectories generally needed a lesser number of arcs, while the longest trajectories required more arcs. The lunar orbits at arrival have eccentricities between $e=0.927$ and $e=0.963$. Figure 8 shows the plots of the time of flight, propellant to initial mass ratio $m_{\mathrm{p}} / m_{0}$, and the height of the flyby with the Moon for all the values of the angle $\theta_{\mathrm{sm} 0}$ considered. Minima can be seen around $\theta_{\mathrm{sm} 0}=0^{\circ}, 180^{\circ}$; and it can be noted that they roughly correspond to the maxima of the $h_{\mathrm{flyby}}$, which
Table 3 Design variable values for the trajectory with $\theta_{\mathrm{sm} 0}=-63^{\circ}$

\begin{tabular}{cccc}
\hline Number of arcs & $\Delta t($ day $)$ & $\alpha(\mathrm{deg})$ & $\beta(\mathrm{deg})$ \\
\hline 1 & 4.112 & -169.676 & 57.919 \\
2 & 14.176 & -71.686 & -22.700 \\
3 & 27.985 & -69.839 & -27.865 \\
4 & 7.112 & -48.758 & -8.150 \\
5 & 9.365 & -43.015 & -24.340 \\
6 & 23.736 & -14.619 & -10.417 \\
7 & 23.755 & 7.479 & -5.122 \\
8 & 64.520 & -11.906 & -54.167 \\
9 & 65.219 & 170.868 & 30.101 \\
10 & 14.117 & 137.646 & 16.012 \\
11 & 54.853 & -163.998 & -35.248 \\
12 & 47.469 & -160.880 & -17.451 \\
13 & 42.336 & -103.778 & -7.758 \\
14 & 38.809 & -55.258 & -12.083 \\
15 & 25.368 & -54.266 & -21.555 \\
16 & 5.182 & 43.908 & 14.801 \\
\hline
\end{tabular}

resulted in variations between 829 and $3200 \mathrm{~km}$.

Figure 9 shows the behavior of the $\Delta V$ with respect to $\theta_{\text {sm0 }}$. The $\Delta V_{\text {traj }}$ is the effective $\Delta V$ exerted during the trajectory, and its behavior is similar to that of $m_{\mathrm{p}} / m_{0}$ and $t_{\mathrm{f}}$, with the same maxima and minima. The $\Delta V_{\text {circ }}$ is the impulsive $\Delta V$ that should be exerted to circularize the lunar orbit at an altitude of $200 \mathrm{~km}$, and it does not represent the true circularization effort - which will need to be carried out with low-thrust propulsion. The only thing worth noting from it is that the orbits achieved are quite similar as the difference in $\Delta V_{\text {circ }}$ between one another is just some tens of $\mathrm{m} / \mathrm{s}$. The $\Delta V_{\text {tot }}$ in the final plot is the sum of the previous two, and includes the departure $\Delta V_{\text {dep }}$ given by the launcher to put the 

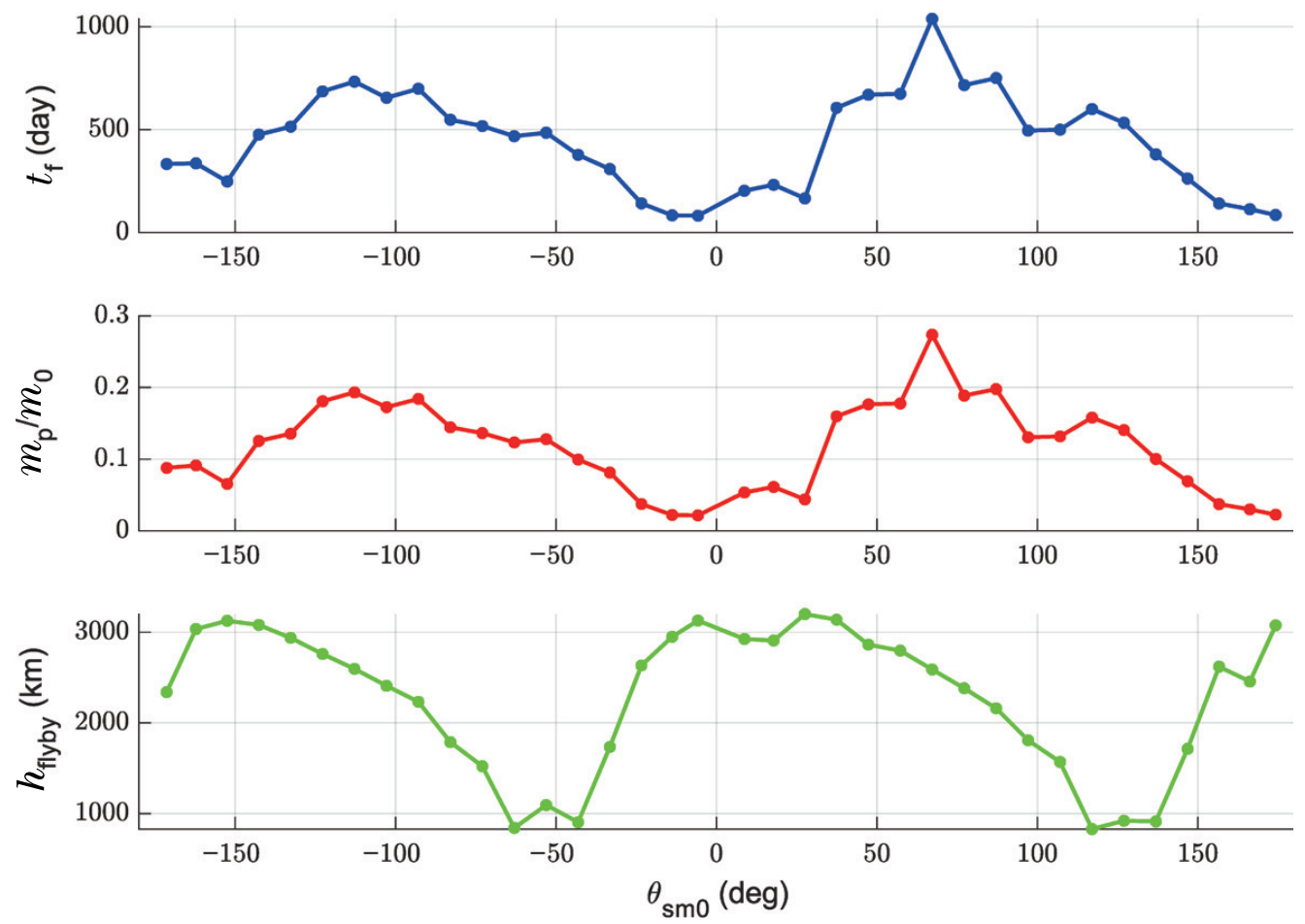

Fig. 8 Time of flight $\left(t_{\mathrm{f}}\right)$, propellant mass ratio $\left(m_{\mathrm{p}} / m_{0}\right)$, and flyby height $\left(h_{\mathrm{flyby}}\right)$.
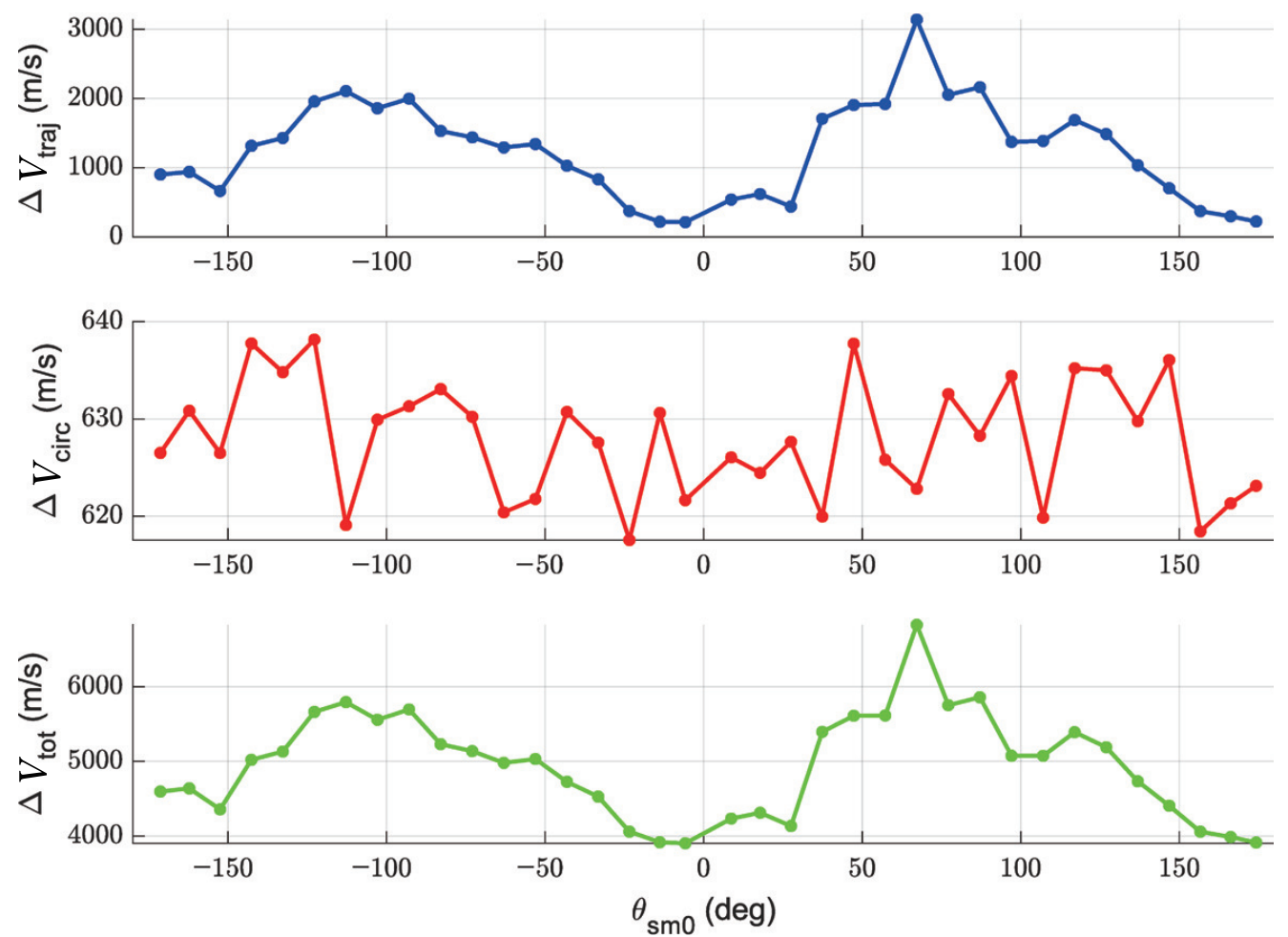

Fig. 9 Trajectory $\Delta V$, Circularization impulsive $\Delta V$, and total $\Delta V$.

payload in lunar transfer from a circular orbit around the Earth - which is estimated to be $3068 \mathrm{~m} / \mathrm{s}$.

In both Fig. 8 and the first and last plots of Fig. 9, it can be seen that the solutions exhibit some periodicity after $180^{\circ}$ of variation of the angle parameter. This behavior is due to the symmetry of the solar gradient around the Earth for variations of $180^{\circ}$ of the Sun's position. If the orbit of the Earth had been considered perfectly 
circular and the perturbation of the Sun approximated for small distances from the Earth, then every trajectory would remain unchanged if the position of the Sun was shifted by $180^{\circ}$. Because there is a small eccentricity of the Earth orbit and the complete perturbation expression is considered, there are differences that require calculating the trajectory even for the almost-symmetric cases; however, it was possible to employ the solutions already computed as a starting guess for the corresponding $180^{\circ}$ shifted cases.

As stated before, the inclinations of the obtained lunar orbits are free, and they result in a uniform distribution between $30^{\circ}$ and $165^{\circ}$. The Horyu mission needs an equatorial orbit around the Moon in order to properly achieve its objective; therefore, a constraint on the inclination of the lunar orbit is considered here. All the trajectories are forced to arrive in the equatorial orbit around the Moon; both prograde and retrograde equatorial orbits are considered valid, and the interval of inclinations allowed is $i \in\left[0^{\circ}, 30^{\circ}\right]$ and $i \in\left[150^{\circ}, 180^{\circ}\right]$. It was possible to achieve the orbits in this range for all trajectories - often at the cost of a higher $t_{\mathrm{f}}$. Figure 10 shows the inclinations before the constraint (upper plot) and after the constraint (lower plot). Figure 11 shows the difference in time of flight, propellant, and $\Delta V$ with respect to the unconstrained trajectories. It can be seen that for most of the trajectories the difference in duration is less than 10 days, and the maximum positive difference is 32.98 days - corresponding to $173.7 \mathrm{~g}$ of extra propellant and a $\Delta V$ of $93.22 \mathrm{~m} / \mathrm{s}$. In one case a notable difference is found: -24.85 days, $-130.9 \mathrm{~g}$, and $-66.69 \mathrm{~m} / \mathrm{s}$.

To provide an outline of the trajectories not shown before, and to visualize how they vary with the variation of the angle parameter, twelve trajectories are plotted in Fig. 12 (one every $30^{\circ}$ ) - around a visualization of the Sun-Earth-Moon angle in which the direction of the Moon is visualized as a blue arrow and the direction of the Sun for all the angles as red arrows. With this disposition, it is easy to see the zones in which typical trajectories appear. The trajectories in this figure include the constraint on the inclination.

\section{Flyby sensitivity and effect of the solar radiation pressure}

The initial flyby of the Moon has a significant influence on the rendezvous trajectory; further, the initial position of the Moon is of great importance in WSB transfers. Here, a brief analysis of the sensitivity of the problem to small variations in the initial position of the Moon is
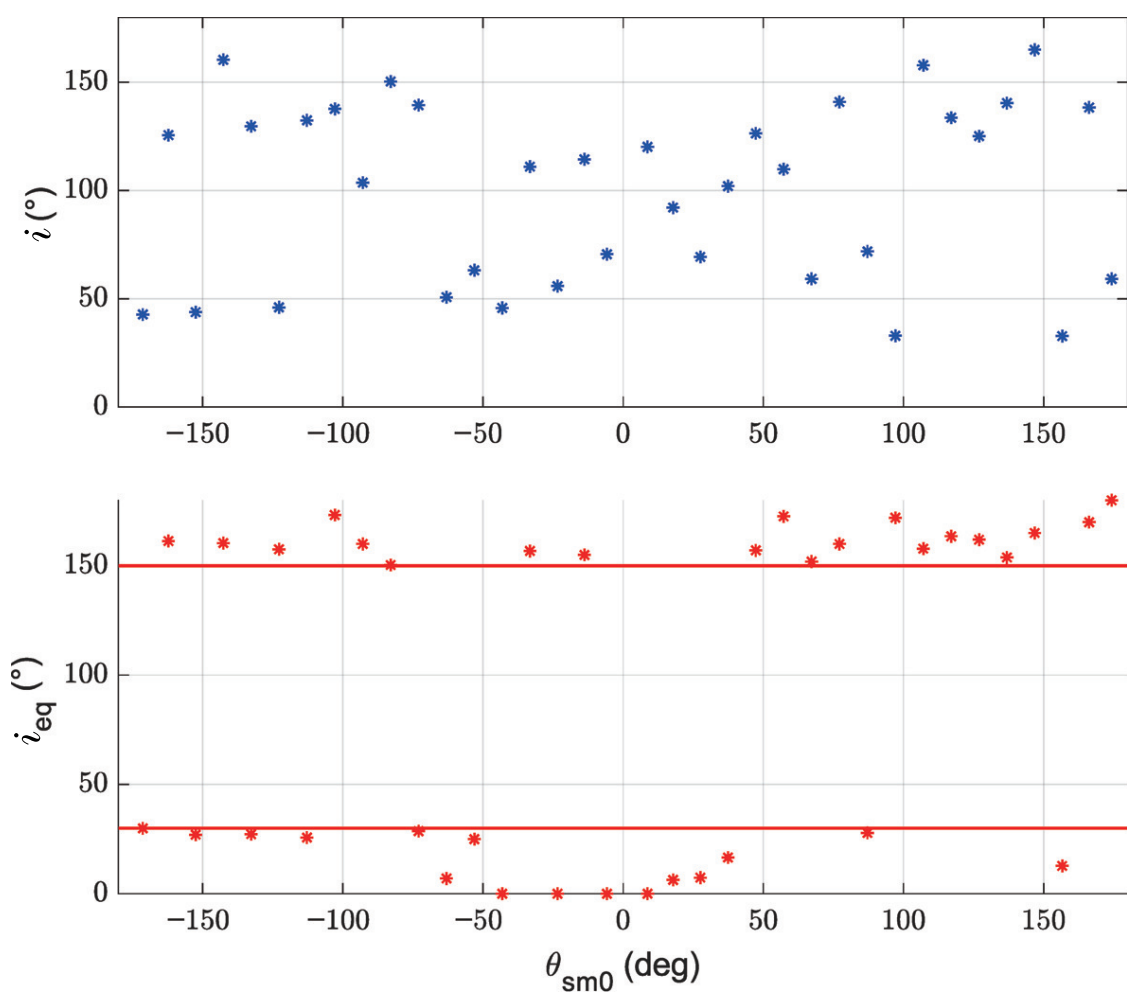

Fig. 10 Lunar inclination with and without equatorial constraint.

\section{(10) TNIVERSTYY PRESA}



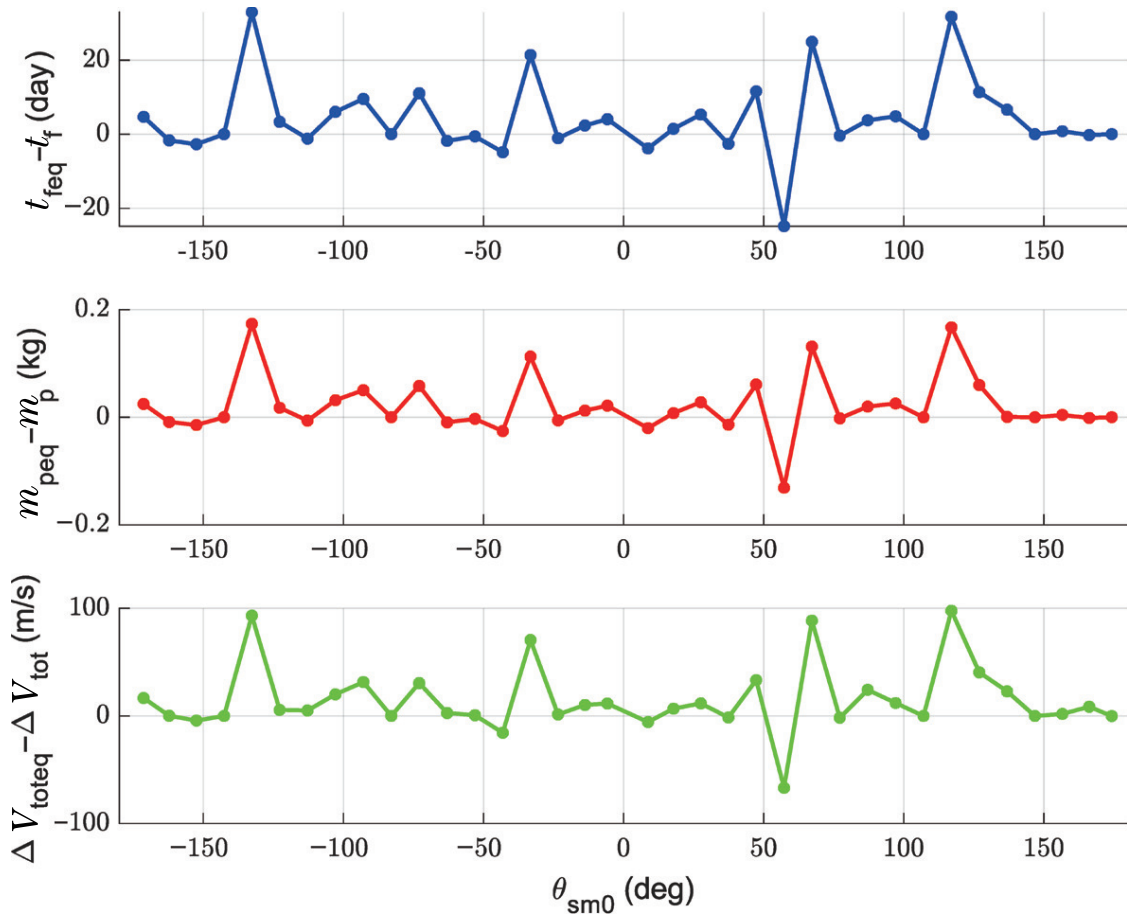

Fig. 11 Differences in time of flight, propellant mass, and total $\Delta V$ between trajectories with equatorial inclination of the final orbit and free trajectories.

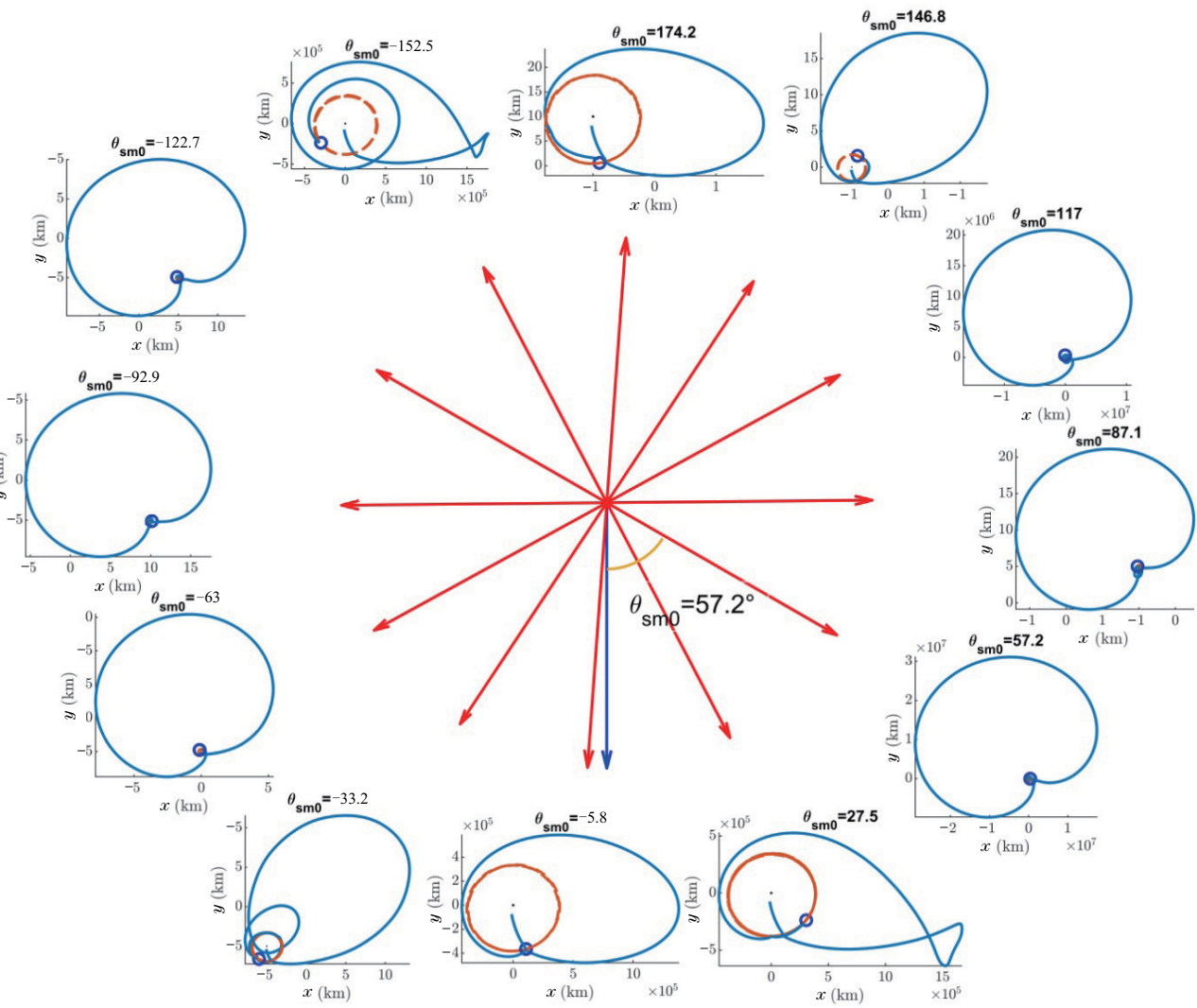

Fig. 12 Outline of the transfer trajectories with the $\theta_{\mathrm{sm} 0}$ parameter. The blue arrow represents the direction of the Moon and the red arrows represent the direction of the Sun at twelve different angles; one angle is highlighted as an example. Trajectories are plotted in the Earth-centered frame. 
presented. The best trajectory among the ones found, in the case where the constraint on the inclination at the arrival at the Moon is considered as a case study; the Moon is moved along its orbit by steps of $1^{\circ}$ both ahead and behind the nominal initial position - then the trajectory is re-optimized to achieve the rendezvous again. With a displacement of $-1^{\circ}$, the performance worsens; the flyby has a lower altitude, and the duration of the trajectory is increased by 28.16 days. With a displacement of $+1^{\circ}$, the performance improved, the flyby height increased, and the duration was reduced by 4.64 days. Continuing the analysis in the favorable direction shows that the initial position displaced by $+1^{\circ}$ is a minimum around the nominal trajectory; in fact, moving to $+2^{\circ}$, the performance is still better - but of a smaller amount. At $+3^{\circ}$ instead, the duration increases, and also at $+4^{\circ}$ even when the shape of the trajectory is changed.

Table 4 summarizes what is said above, showing the difference in duration, propellant, flyby height, and apogee with respect to the nominal trajectory. Figure 13 shows the trajectories found for every position variation and also the nominal trajectory $\left(0^{\circ}\right)$ for comparison.

In the analysis in the previous section, the effect of the solar radiation pressure was not taken into account; here, we consider its effect on the best trajectory in a simplified way. The area of the spacecraft is considered constant during the trajectory, equal to the maximum area of the satellite and always facing the direction of the Sun.
Table 4 Results of the variation of the initial position of the Moon

\begin{tabular}{cllll}
\hline Moon displ. & $\begin{array}{c}\delta t_{\mathrm{f}} \\
(\text { day })\end{array}$ & $\begin{array}{c}\delta m_{\mathrm{p}} \\
(\mathrm{g})\end{array}$ & $\begin{array}{c}\delta h_{\mathrm{flyby}} \\
(\mathrm{km})\end{array}$ & $\begin{array}{c}\delta r_{\text {apo }} \\
\left(10^{5} \mathrm{~km}\right)\end{array}$ \\
\hline$-1^{\circ}$ & +28.16 & +148.4 & -979.7 & +4.181 \\
$+1^{\circ}$ & -4.64 & -24.5 & +891.7 & -0.706 \\
$+2^{\circ}$ & -4.08 & -21.5 & +1242.1 & -0.430 \\
$+3^{\circ}$ & +2.40 & +12.6 & +1490.6 & -0.481 \\
$+4^{\circ}$ & +37.84 & +19.9 & +3211.0 & -4.175 \\
\hline
\end{tabular}

Full specular reflection is considered in such a way that the acceleration given by the solar radiation pressure is always in the opposite direction of the Sun. These conditions allow an estimate of the maximum possible effect of the SRP on the trajectory.

$$
\boldsymbol{a}_{\mathrm{SRP}}=P_{\mathrm{E}} \frac{A}{m} \widehat{\boldsymbol{r}}_{\mathrm{s}}
$$

Equation (10) shows the expression of the acceleration of the SRP in the considered conditions; $P_{\mathrm{E}}$ is the solar radiation pressure at $1 \mathrm{AU}, A$ is the area of the spacecraft, $m$ is the mass, and $\widehat{\boldsymbol{r}}_{\mathrm{s}}$ is the unit vector from the Sun to the spacecraft. The numerical results show that the solar radiation pressure has a negligible effect and the nominal trajectory has its duration changed by $2600 \mathrm{~s}$.

\section{Stabilization and circularization}

In this section, the problem of obtaining a stable orbit around the Moon is addressed. The trajectory with the
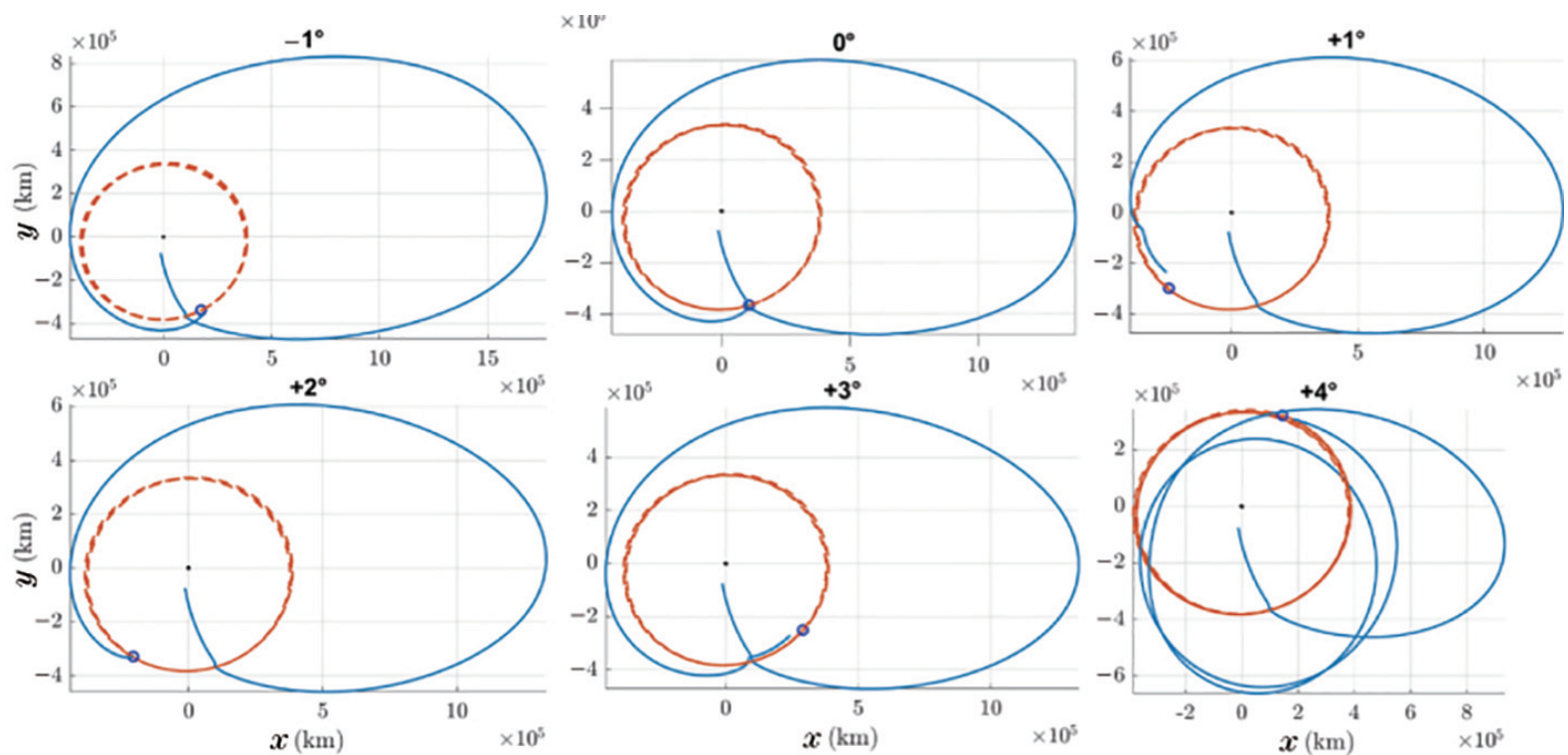

Fig. 13 Transfer trajectory with $\theta_{\mathrm{sm} 0}=-5.78^{\circ}$ subject to small variations of the initial position of the Moon. Plots in the Earth-centred inertial reference frame.

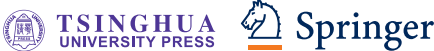


least time of flight among the previous trajectories was considered as a case study.

The final semi-axis and eccentricity of the lunar rendezvous trajectory are $a=29,571 \mathrm{~km}, e=0.934$. If left free, the spacecraft will quickly escape from the Moon; instead, the spacecraft must achieve a circular orbit at an altitude of $100 \mathrm{~km}$. For this purpose, the trajectory is continued with some arcs in which the direction of the thrust is constrained to vary in a cone around the anti-velocity direction; an optimization is run with the objective of reducing the semi-axis below $20,000 \mathrm{~km}$ and the eccentricity below 0.7 , in order to reach a stable orbit. Then, the orbit is circularized, and the height is reduced by employing the following strategy:

- First, the thrust is in the anti-velocity direction during the orbit when the true anomaly is inside an interval centered around the pericenter, here taken as $\left[-130^{\circ}, 130^{\circ}\right]$. When this condition is not met, the thruster is shut off. This reduces the semi-axis and eccentricity of the orbit.

- After the eccentricity is reduced to almost zero, the thrust returns continuously during all the orbits in the anti-velocity direction until the required height is reached.

Table 5 shows the duration and propellant consumption of the considered trajectory; in the first two columns, the time and propellant are divided between the transfer trajectory (without the added arcs used for stabilization) and the stabilization and circularization maneuver until the final orbit is reached. The last column presents the total cost of the mission. The propellant needed is inside the $3 \mathrm{~kg}$ limit with a margin of $0.4928 \mathrm{~kg}$, and the total duration is 765.77 days, of which 484.75 days are of thrusting. Figure 14 shows the trajectory from the arrival around the Moon to the end of the maneuver on the final orbit; and Fig. 15 shows the evolution of the orbital elements during stabilization. To be more readable, the plot starts from 81.54 days after departure from the Earth, when the original transfer trajectory is ended. The semi-axis is gradually reduced and the eccentricity is subject to some oscillations during the initial phase, before becoming more stable and starting to decrease until it reaches zero. The inclination of the orbit reached is $11^{\circ}$ - it is therefore acceptable for the imposed constraints.

As a final remark, if instead of a low orbit at an altitude of $100 \mathrm{~km}$, the requirements had been to simply place the
Table 5 Cost of the trajectory from the Earth to low lunar orbit

\begin{tabular}{cccc}
\hline & $\begin{array}{c}\text { Transfer trajectory } \\
\left(\theta_{\mathrm{sm} 0}=-5.78^{\circ}\right)\end{array}$ & $\begin{array}{c}\text { Stabilization and } \\
\text { circularization }\end{array}$ & Total \\
\hline$t$ (day) & 81.54 & 684.23 & 765.77 \\
$m_{\mathrm{p}}(\mathrm{kg})$ & 0.4297 & 2.0775 & 2.5072 \\
\hline
\end{tabular}

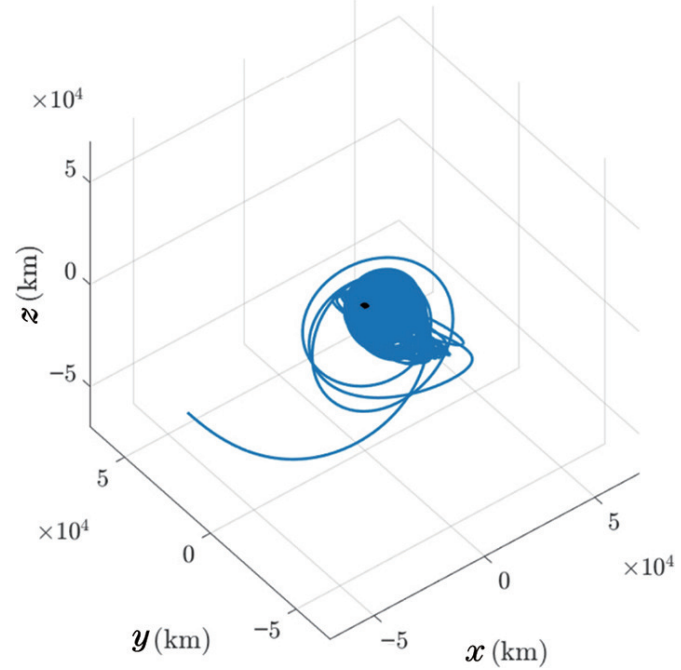

Fig. 14 Trajectory to low lunar orbit, plotted in the Mooncentric inertial frame.
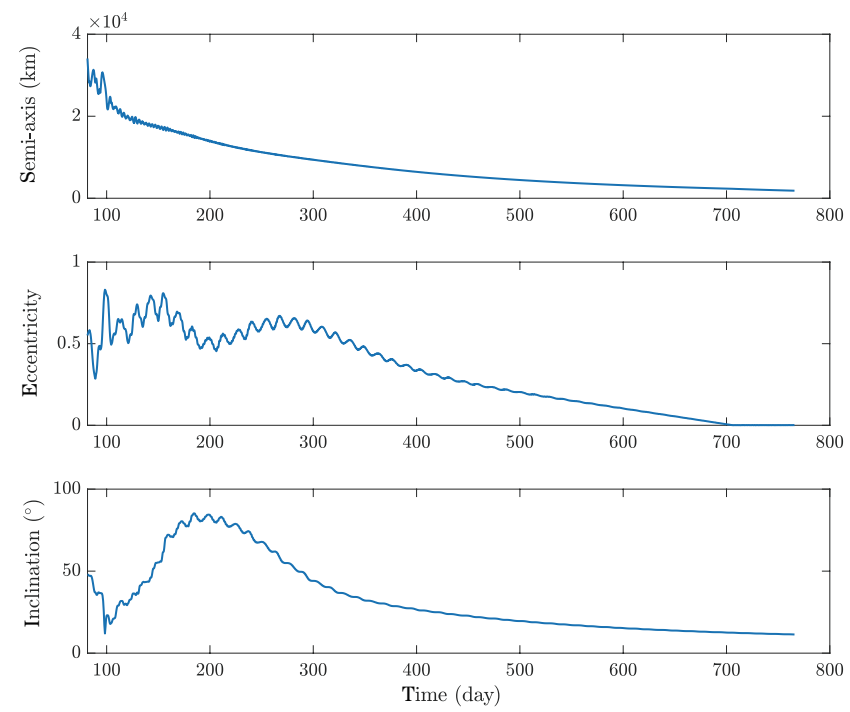

Fig. 15 Orbital elements during the stabilization and circularization trajectory.

spacecraft in a stable orbit, then clearly the total cost would have been notably less. The trajectory presented in this section can be taken as an example; if it is truncated at 37.23 days after the end of the Earth-Moon transfer, it yields an orbit with a semi-axis of about 19,000 km (having a pericenter height of $6400 \mathrm{~km}$, and an apocenter of $28,800 \mathrm{~km}$ ) that remains stable for at least 500 days. 
The propellant cost would be $0.2 \mathrm{~kg}$ over the transfer trajectory cost, so the total cost would be $0.626 \mathrm{~kg}$ about a quarter of the mass to reach the $100 \mathrm{~km}$ circular orbit.

\section{Conclusions}

This work presented the trajectory analysis of the HoryuVI nano-satellite, an international lunar mission with the goal of studying the lunar horizon glow.

In particular, the effect of the solar gravity gradient on the trajectory was analyzed, covering the entire range of values of the Sun-Earth-Moon angle at departure. With the given low-thrust propulsion system, a transfer trajectory was found for all configurations, achieving a lunar orbit with eccentricity less than one, and a pericenter at an altitude of $200 \mathrm{~km}$. The range of propellant required varies from $490 \mathrm{~g}$ to $5.47 \mathrm{~kg}$, although the most expensive cases require a greater quantity of propellant than the expected $3 \mathrm{~kg}$. It was found that the best trajectories resemble the WSB Earth-Moon transfers in the way they exploit the Sun gradient to decelerate and accelerate where most needed; therefore, these types of trajectories should be considered the most feasible for the considered problem.

A constraint on the inclination of the arrival orbit at the Moon is also considered, and the difference in cost and time is computed with respect to the unconstrained case. Reaching an equatorial orbit is possible in all configurations, and the differences in time and propellant are small.

Among the transfer trajectories, some further analysis was performed on the best one considering its sensitivity to small changes in the Moon's initial position-which affect the flyby. It was found that a few degrees of variation in the angular position are admissible without drastic changes in the trajectory - and in some cases, they might even produce an improvement in the performance. It was also assessed that the solar radiation pressure should be of very little importance for this mission.

The problem of the stabilization of the lunar orbit was also considered. In the specific case of the best transfer trajectory, the cost of this maneuver is higher than the transfer trajectory cost. Reaching the final low lunar orbit of the Horyu-VI mission required a total of $2.5 \mathrm{~kg}$ of propellant - equal to $12.5 \%$ of the initial mass. These results show that with the given allocated propellant mass, and considering the best transfer trajectory, the mission results are feasible.

\section{Funding note}

Open Access funding provided by Università degli Studi di Roma La Sapienza.

\section{References}

[1] Örger, N. C., Cho, M., Iskender, O. B., Lim, W. S., Chandran, A., Ling, K. V., Holden, K. H., Chow, C. L., Bellardo, J., Faure, P., et al. Horyu-VI: International CubeSat mission to investigate lunar horizon glow. In: Proceedings of the 71st International Astronautical Congress, 2020: IAC-20,B4,2,7,x55547.

[2] Barker, M. K., Mazarico, E., McClanahan, T. P., Sun, X., Neumann, G. A., Smith, D. E., Zuber, M. T., Head, J. W. Searching for lunar horizon glow with the lunar orbiter laser altimeter. Journal of Geophysical Research: Planets, 2019, 124(11): 2728-2744.

[3] Colwell, J. E., Batiste, S., Horanyi, M., Robertson, S., Sture, S. Lunar surface: Dust dynamics and regolith mechanics. Reviews of Geophysics, 2007, 45(2): 2005RG000184.

[4] Criswell, D. R. Horizon-glow and the motion of lunar dust. In: Photon and Particle Interactions with Surfaces in Space. Grard, R. J. L. Ed. Dordrecht: Springer, 1973: 545-556.

[5] Feldman, P. D., Glenar, D. A., Stubbs, T. J., Retherford, K. D., Randall Gladstone, G., Miles, P. F., Greathouse, T. K., Kaufmann, D. E., Parker, J. W., Alan Stern, S. Upper limits for a lunar dust exosphere from farultraviolet spectroscopy by LRO/LAMP. Icarus, 2014, 233: 106-113.

[6] Glenar, D. A., Stubbs, T. J., Hahn, J. M., Wang, Y. Search for a high-altitude lunar dust exosphere using clementine navigational star tracker measurements. Journal of Geophysical Research: Planets, 2014, 119(12): 2548-2567.

[7] Glenar, D. A., Stubbs, T. J., McCoy, J. E., Vondrak, R. R. A reanalysis of the apollo light scattering observations, and implications for lunar exospheric dust. Planetary and Space Science, 2011, 59(14): 1695-1707.

[8] McCoy, J. E. Photometric studies of light scattering above the lunar terminator from apollo solar corona photography. In: Proceedings of the Lunar and Planetary Science Conference, 1976, 7: 1087-1112.

[9] McCoy, J. E., Criswell, D. R. Evidence for a high altitude distribution of lunar dust. In: Proceedings of Lunar and Planetary Science Conference, 1974, 5: 2991-3005. 
[10] Rennilson, J. J., Criswell, D. R. Surveyor observations of lunar horizon-glow. The Moon, 1974, 10(2): 121-142.

[11] Severny, A. B., Terez, E. I., Zvereva, A. M. The measurements of sky brightness on lunokhod-2. The Moon, 1975, 14(1): 123-128.

[12] Zook, H. A., McCoy, J. E. Large scale lunar horizon glow and a high altitude lunar dust exosphere. Geophysical Research Letters, 1991, 18(11): 2117-2120.

[13] Gaier, J. R. The effects of lunar dust on EVA systems during the Apollo missions. Report No. E-15071. NASA Glenn Research Center, Cleveland OH, 2005.

[14] Harris, R. S. Jr. Apollo experience report: Thermal design of Apollo lunar surface experiments package. Report No. TN D-6738. National Aeronautics and Space Administration, WDC, 1972.

[15] James, J. T., Lam, C. W., Quan, C., Wallace, W. T., Taylor, L. Pulmonary toxicity of lunar highland dust. SAE Technical Paper 2009-01-2379, 2009, https://doi.org/10.4271/2009-01-2379.

[16] Linnarsson, D., Carpenter, J., Fubini, B., Gerde, P., Karlsson, L. L, Loftus, D. J., Prisk, G. K., Staufer, U., Tranfield, E. M., van Westrenen, W. Toxicity of lunar dust. Planetary and Space Science, 2012, 74(1): 57-71.

[17] O'Brien, B. J. Review of measurements of dust movements on the Moon during Apollo. Planetary and Space Science, 2011, 59(14): 1708-1726.

[18] O'Brien, B. J. Paradigm shifts about dust on the Moon: From Apollo 11 to Chang'e-4. Planetary and Space Science, 2018, 156: 47-56.

[19] Niccolai, L., Bassetto, M., Quarta, A., Mengali, G. A review of Smart Dust architecture, dynamics, and mission application. Progress in Aerospace Sciences, 2019, 106: $1-14$.

[20] Smith, D. A. Space Launch System (SLS) Mission Planner's Guide. NASA M19-7163, 2018.

[21] Del Monte, M., Meis, R., Circi, C. Optimization of interplanetary trajectories using the colliding bodies optimization algorithm. International Journal of Aerospace Engineering, 2020, 2020: 9437378.

[22] Belbruno, E., Miller, J. Sun-perturbed Earth-to-Moon transfers with ballistic capture. Journal of Guidance, Control, and Dynamics, 1993, 16: 770-775.

[23] Kawaguchi, J., Yamakawa, H., Uesugi, T., Matsuo, H. On making use of lunar and solar gravity assist for Lunar A and planet B missions. Acta Astronautica, 1995, 35: 633-642.

[24] Romagnoli, D., Circi, C. Earth-Moon weak stability boundaries in the restricted three and four body problem. Celestial Mechanics and Dynamical Astronomy, 2009, 103(1): 79-103.

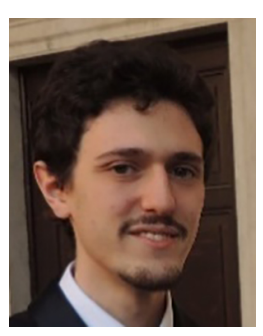

Federico De Grossi received his M.S. degree in space and astronautical engineering at Sapienza University of Rome in 2019, with a thesis on rendezvous trajectories with orbits around asteroids. He is currently a Ph.D. student, and his fields of study are space mission trajectory analysis and optimization, optimization methods, and quantum information and computation. E-mail: federico.degrossi@uniroma1.it.

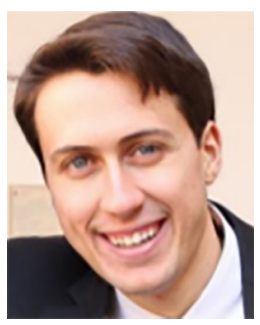

Paolo Marzioli is a post-doctoral research fellow at Sapienza University of Rome. He received his Ph.D. degree in aeronautical and space engineering in 2021, with a thesis on the implementation of navigation systems for nano-satellites. His main research topics deal with nanosatellite missions, stratospheric experiment development and tracking, and navigation systems evaluation for different aerospace mission profiles. E-mail: paolo.marzioli@uniroma1.it.

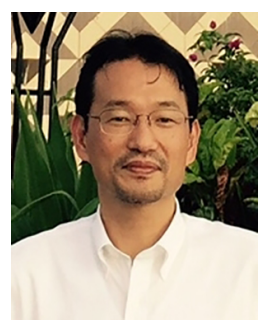

Mengu Cho received his B.S. and M.S. degrees from the University of Tokyo, and his Ph.D. degree from Massachusetts Institute of Technology, in 1992. After working at Kobe University and International Space University, he joined Kyushu Institute of Technology (Kyutech) in 1996. Since 2004, he has been a professor. Currently, he is the director of Laboratory of Lean Satellite Enterprises and In-Orbit Experiments. His research interests include spacecraft environmental interaction and satellite systems. He has supervised 11 university satellite projects, among which 9 projects and 16 satellites have already been launched. He has authored or co-authored more than 180 papers in peer reviewed journals. His research interest is nano-/micro-satellite development and applications. In 2019, he received Frank J. Malina Astronautics Medal from International Astronautical Federation. E-mail: cho.mengu801@gmail.kyutech.jp. 


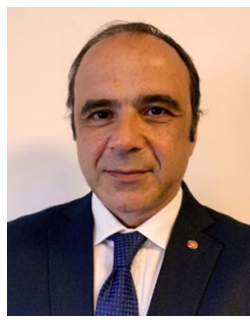

Fabio Santoni is an associate professor of aerospace systems at Sapienza University of Rome. His main research topics are nano-satellite mission design, development and operations, space debris observations and mitigation, and sustainable space exploration. E-mail: fabio.santoni@uniroma1.it.

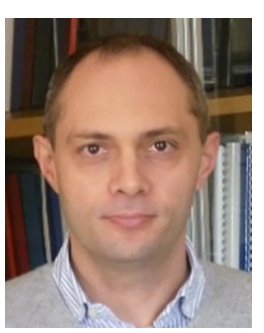

Christian Circi is currently an associate professor in flight mechanics at the Department of Astronautical, Electrical, and Energy Engineering, Sapienza University of Rome. He received his M.S. degree in aeronautical engineering and aerospace engineering and his Ph.D. degree in aerospace engineering at Sapienza

University of Rome. He worked as a researcher at the Grupo de Mecanica of Vuelo-Madrid (GMV) and a research assistant at the Department of Aerospace Engineering. $\mathrm{He}$ is a lecturer in "Interplanetary Trajectories" and "Flight Mechanics of Launcher" in the master degree course of space and astronautical engineering at Sapienza University of Rome. His principal research fields are third-body and solar perturbations, interplanetary and lunar trajectories, so- lar sails, orbits for planetary observation, and the ascent trajectory of Launcher. He is the associate editor for Aerospace Science and Technology and the International Journal of Aerospace Engineering. E-mail: christian.circi@uniroma1.it.

Open Access This article is licensed under a Creative Commons Attribution 4.0 International License, which permits use, sharing, adaptation, distribution and reproduction in any medium or format, as long as you give appropriate credit to the original author(s) and the source, provide a link to the Creative Commons licence, and indicate if changes were made.

The images or other third party material in this article are included in the article's Creative Commons licence, unless indicated otherwise in a credit line to the material. If material is not included in the article's Creative Commons licence and your intended use is not permitted by statutory regulation or exceeds the permitted use, you will need to obtain permission directly from the copyright holder.

To view a copy of this licence, visit http://creativecommons.org/licenses/by/4.0/. 